\title{
P I 4-02. Social impact events in Phambili, the first phase 2B HIV vaccine trial in South Africa
} MA Allen*1,5, G Gray², G Churchyard ${ }^{3}$, L Bekker ${ }^{4}$, M Nchabeleng8, K Mlisana $^{6}$, B Metch 7 , Z Moodie ${ }^{7}, \mathrm{~S} \mathrm{Roux}^{4}, \mathrm{M} \mathrm{Mathebula}^{8}, \mathrm{~T}$ Bennie ${ }^{6}$ and J Kublin ${ }^{9}$

\begin{abstract}
Address: ${ }^{1}$ DAIDS/VRP, National Institute of Allergy and Infectious Diseases, NIH, Bethesda, MD, USA, ${ }^{2}$ Perinatal HIV Research Unit and University of Witwatersrand, Soweto, South Africa, ${ }^{3}$ Aurum Institute for Health Research, and University of Cape Town, Klerksdorp, South Africa, ${ }^{4}$ Desmond Tutu HIV Centre, Cape Town, South Africa, ${ }^{5}$ Medunsa Clinical Research Unit (MeCRU), Medunsa, South Africa, ${ }^{6}$ Centre for the AIDS Programme for Research in South Africa (CAPRISA), Durban, South Africa, ${ }^{7}$ Statistical Center for HIV/AIDS Research and Prevention, FHCRC, Seattle, WA, USA, ${ }^{8}$ Medunsa Clinical Research Unit (MeCRU), Medunsa, South Africa and ${ }^{9}$ HIV Vaccine Trials Network, Fred Hutchinson Cancer Research Center, Seattle, WA, USA

* Corresponding author
\end{abstract}

from AIDS Vaccine 2009

Paris, France. 19-22 October 2009

Published: 22 October 2009

Retrovirology 2009, 6(Suppl 3):PI90 doi:10.1186/1742-4690-6-S3-PI90

This abstract is available from: http://www.retrovirology.com/content/6/S3/PI90

(c) 2009 Allen et al; licensee BioMed Central Ltd.

\section{Background}

Participants in preventive HIV vaccine trials may experience social impact events (SIEs) related to study including stigmatization and problems due to positive HIV tests from vaccine-induced HIV antibody. Data are presented on SIEs in the first Phase 2B HIV vaccine trial in South

\section{Methods}

From January 2007 through April 2009, 801 participants enrolled in Phambili (HVTN 503) underwent standardized assessments for SIE at scheduled time-points as well as whenever reported by participants off-schedule.

\section{Results}

Forty six $(5.7 \%)$ of study participants (28 women, 18 men) reported 51 SIEs. Most commonly reported (n 46, $90 \%$ ) were negative reactions of family, friends, and coworkers to the volunteer's study participation. Few events pertained to employment (n 2), medical/dental care (n 2), or other categories. No participants reported problems with education, housing, military service, travel/immigration, or health/life insurance. HIV testing was performed outside of the study in relation to 2 events (health-care related).
Most events followed participants' voluntary disclosure of study participation (n 29, 56.9\%), although in many events ( $\mathrm{n} 15,29.4 \%$ ), study participation was not revealed.

Participants assessed most events $(74.5 \%)$ as minimal impact on quality of life (QOL), and the majority (58.5\%) as resolved. Of the 5 events with major impact on QOL, 3 involved personal relationships, 1 employment, and 1 other cause; none were resolved despite participant and staff efforts. The majority of events (n 28, 55\%) were reported within the first month of enrollment, prior to the scheduled assessment at month 3. Some events occurred during screening.

\section{Conclusion}

Incidence of reporting was relatively low, with very few major events reported. The majority of events were reported very early in study.

Informing potential study participants about the risk of SIE, and providing participants with assistance to prevent or resolve events may be effective strategies in reducing incidence and severity of SIEs. 